\title{
Comment on the paper
}

\section{"Definition of the river Gacka springs subcatchment areas on the basis of hydrogeological parameters" by Lukač Reberski et al., published in Geologica Croatica 66/1, 39-53 (2013)}

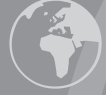

Zvjezdana Roller-Lutz ${ }^{1}$, Tamara Hunjak ${ }^{1}$ and Hans O. Lutz ${ }^{1,2}$

${ }^{1}$ Medical Faculty, Rijeka University, Braće Branchetta 20, 51000 Rijeka, Croatia; (roller@medri.uniri.hr) ${ }^{2}$ Faculty of Physics, Bielefeld University, Bielefeld, Germany
\end{abstract}

doi: 10.4154/gc.2013.12

\section{Geologia-Groatice.}

This paper by Lukač Reberski et al. (in the following referred to as paper I) presents measurements of hydrological and hydrochemical parameters as well as results of an isotopic analysis of the spring waters and the precipitation. As the authors of paper I mention, the isotopic analysis has been performed in Graz and in Rijeka. We want to point out that the data for the year 2008 resulted from a cooperation project which was headed by Rijeka University and supported by the International Atomic Energy Agency (IAEA Vienna); the corresponding isotope results were preliminary and shown only in an unpublished and not citable report. In spite of a confidentiality agreement, the authors of paper I took these data without our consent. This breach of trust and the somewhat sloppy data handling has some unfortunate consequences:

The isotope data shown in Fig.7 appear to scatter between the Local and the Global Meteoric Water Lines, LMWL and GMWL. This was used to argue a direct correspondence between precipitation and spring recharge. Firstly, note that this LMWL is plotted incorrectly in Fig. 7; the correct LMWL (eq.5 in paper I) had to be lower by about -1\%o (i.e. shifted to more negative $\delta 2 \mathrm{H}$ values) if compared to the plotted line. Therefore, the majority of data points are actually located slightly above the correct LMWL. The final analysis of the 2008 isotopic data further corroborates this small but noticeable effect; as a consequence, the d-excess $\mathrm{d}=\delta 2 \mathrm{H}$ $-8 \cdot \delta 180$ is somewhat higher for the spring waters than that of the precipitation, supporting the conclusion that recharge happens mainly during the cold season (c.f. also MANDIĆ et al. (2008)).
Furthermore, the absolute altitude values of the recharge areas (c.f. the end of section 7.3 in paper I) cannot simply be inferred from the measured isotopic ratios; the available data can only provide (approximate) values for the altitude differences. The reason is that the derivation of absolute values requires some reference data, e.g. the long-term mean $\delta 180$ of the precipitation at the spring altitude (more precisely, taking into account the mean residence time of the ground water as well as the infiltration into the catchment); or a reliable value of the absolute altitude of at least one of the recharge areas (assuming that the soil surface and thus the infiltration is of approximately the same nature for all catchments). Without further discussion neither is really known; for example, depending on the season, the $\delta 180$ values of the collected and analyzed rainfall in the Gacka spring region in the years 2006 and 2008 vary between approximately $-4 \%$ and $-13 \%$. The authors do not provide any clue for the reference data they used.

The publication of the final analysis of our results, together with an estimate of the mean residence time of the ground water as obtained from tritium model calculation, is presently under preparation.

\section{REFERENCES}

MANDIĆ, M., BOJIĆ, D., ROLLER-LUTZ, Z., LUTZ, H.O., KRAJCAR BRONIĆ, I. (2008): Note on the spring region of the Gacka River (Croatia).- Isotop. Envir. Health Studies, 44/2, 201-208. 
